\title{
Diseño de un control difuso tipo Mamdani para regular la temperatura de una Acuario tipo tropical
}

\section{Designing a Mamdani type fuzzy control to regulate the temperature of a tropical Aquarium}

\author{
DE LA CRUZ-ALEJO, Jesús†* \& LOYA-ESCALANTE, María Teresa
}

Tecnológico de Estudios Superiores de Ecatepec

ID $1^{\text {er }}$ Autor: Jesús, De La Cruz-Alejo / ORC ID: 0000-0001-5072-3985, CVU CONACYT ID: 173289

ID $1^{\text {er }}$ Coautor: María Teresa, Loya-Escalante / ORC ID: 0000-0003-1515-4312, CVU CONACYT ID: 635849

DOI: $10.35429 / J I D .2019 .8 .3 .1 .9$

Recibido 09 Julio, 2019; Aceptado 03 de Septiembre, 2019

Resumen

En el presente trabajo de investigación, se muestra una técnica de control automático basada en lógica difusa para regular las condiciones de temperatura en un acuario tropical con capacidad para 100 litros. Para analizar la efectividad de la técnica se realizó el diseño y la implementación de un controlador de lógica difusa tipo Mamdani. Posteriormente el controlador difuso se integró a un sistema eléctrico utilizando una resistencia de 100 watts. La regulación se realiza a partir de la adquisición de la temperatura del agua, variando así la corriente de la resistencia, se utilizó un sensor de temperatura, un calentador como actuadores. La funcionalidad del sistema se verifica a través de la plataforma Artix-7 FPGA, empleando como lenguaje de descripción de hardware VHDL. Se desarrolló el diseño del controlador mediante la herramienta de fuzzy logic en Matlab para poder comparar resultados experimentales de la FPGA, obteniéndose un error de $2.94 \%$ de exactitud entre ambos diseños.

\section{Lógica difusa, Vhdl, Temperatura}

\begin{abstract}
In the present research work, an automatic control technique based on fuzzy logic is shown to regulate the temperature conditions in a tropical aquarium with a capacity of 100 liters. To analyze the effectiveness of the technique, the design and implementation of a fuzzy logic controller type Mamdani was carried out. Subsequently, the diffuse controller was integrated into an electrical system using a 100-watt resistor. The regulation is carried out from the acquisition of the water temperature, thus varying the current of the resistance, a temperature sensor, and a heater as actuators were used. The functionality of the system is verified through the Artix-7 FPGA platform, using the VHDL hardware description language. The controller design was developed using the fuzzy logic tool in Matlab in order to compare experimental results of the FPGA, obtaining an error of $2.94 \%$ accuracy between both designs.
\end{abstract}

Fuzzy logic, Vhdl, Temperature

Citación: DE LA CRUZ-ALEJO, Jesús \& LOYA-ESCALANTE, Maria Teresa. Diseño de un control difuso tipo Mamdani para regular la temperatura de una Acuario tipo tropical. Revista del Diseño Innovativo. 2019 3-8: 1-9

\footnotetext{
* Correspondencia del Autor (Correo electrónico: jdelacruz@ tese.edu.mx)

$\dagger$ Investigador contribuyendo como primer autor.
} 


\section{Introducción}

Los acuarios son objetos de cristal donde se reproducen los ambientes subacuáticos de agua dulce, salada entre otros, en los que se trata de mantener las condiciones del ecosistema con base al tipo de acuario que se quiera reproducir. Por lo general, se utilizan en hogares, empresas, hoteles con fines ornamentales. Sin embargo, el hecho de tener un acuario requiere llevar a cabo un cuidadoso mantenimiento para que esté en condiciones ideales. Las dimensiones del acuario, tipo de peces que se introducirán dentro del acuario, niveles de oxigenación, temperatura e iluminación son algunas de las condiciones que se deben de tener en cuenta para mantener un acuario en condiciones ambientales óptimas. La temperatura juega un papel importante dentro de las condiciones del ecosistema para los peces que se introducirán, ya que los cambios de temperatura suelen ser críticos en la reproducción de ciertas especies, así también, algunas enfermedades comunes en peces dependen también de la temperatura. [I] [II]

Un factor fisiológico vital para los peces es el metabolismo, y el ritmo del metabolismo es determinado ampliamente por la temperatura del agua ya que la temperatura interna del pez es igual a la temperatura externa del agua. El agua más caliente acelera el ritmo de la digestión y otros procesos biológicos. Esto ocasiona que el pez llegue a la adultez más rápido, pero también acorta su periodo de vida [III] [IV]. Otros procesos biológicos como la eclosión de huevos y desarrollo de los alevines también se ven alterados por una temperatura del agua más elevada.

La lógica difusa no es la respuesta para todos los problemas técnicos, pero si para aquellos problemas de control donde la simplicidad y la rapidez en la implementación son importantes. Algunas de las aplicaciones donde se ha utilizado la lógica difusa es en el control de ambientes, equipos para el hogar, equipos de electrónica y sistemas de automóviles [V] [VI]. Así la lógica difusa tiene una ventaja sobre un sistema de control convencional, el cual, es capaz de incorporar la experiencia de un experto en el área de conocimiento. Esta experiencia es trasladada hacia el diseño de las reglas que definirán las variables lingüísticas que permitan definir las acciones de control dentro del diseño.
El objetivo de este trabajo es diseñar e implementar un sistema de control utilizando lógica difusa tipo Mamdani para regular la temperatura de un acuario bajo un dispositivo lógico programable FPGA.

La aportación del presente trabajo de investigación presenta una alternativa de solución para el control de este tipo de acuarios que permitan disminuir costos, complejidad, eficiencia y bajo consumo de potencia, así como poder generar una base de conocimiento que se puede ir ampliando para proyectos más grandes o donde el control de temperatura sea un factor importante dentro del proceso. El presente trabajo está organizado en las siguientes secciones. Sección 2, se describe el control lógico difuso, sección 3, diseño e implementación del FLC, sección 4, Se realiza la caracterización del sistema, mientras que en la sección 5, se presentan los resultados experimentales del FLC, sección 6, conclusiones son presentadas.

\section{Controlador Lógico Difuso (CLD)}

El diagrama a bloques del controlador propuesto para el control de la temperatura, se presenta en la figura 1, en donde se muestra las etapas del controlador difuso tipo Mamdani. La etapa de pre procesamiento (entrada) se encarga de acondicionar las señales de entrada, puesto que la tarjeta cuenta con entradas analógicasdigitales. En la etapa de Fusificación se convierte la magnitud de la señal en una cantidad difusa, obteniendo el valor de pertenencia que tiene en cada uno de los valores lingüísticos en el universo de discusion.

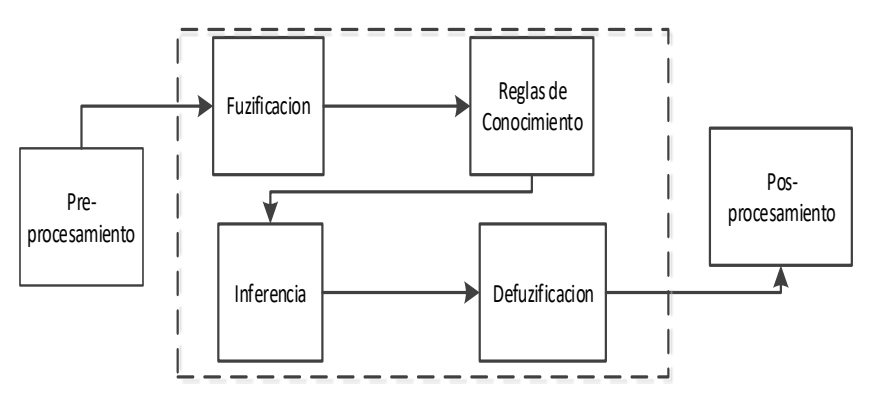

Figura 1 Diagrama del Controlador tipo Mamdani. Fuente: Elaboración Propia

El diseño del controlador propuesto se describe en la tabla 1, donde se puede observar que contiene 2 entradas y una salida, cada entrada cuenta con 5 conjuntos difusos, con una resolución de 10 bits para cada universo de discusión.

DE LA CRUZ-ALEJO, Jesús \& LOYA-ESCALANTE, Maria Teresa. Diseño de un control difuso tipo Mamdani para regular la temperatura de una Acuario tipo tropical. Revista del Diseño Innovativo. 2019 
La propuesta se define de esta manera, al utilizar un mayor número de conjuntos, se tiene una mayor resolución, sin embargo se observó a través de simulaciones realizadas que al utilizar más de 7 conjuntos difusos no aumenta significativamente la eficiencia del controlador y el error de aproximación es casi el mismo, por lo que se concluye que después de 7 conjuntos difusos, el error de aproximación es prácticamente cero y por otro lado, se aumenta la complejidad y el consumo de recursos del sistema por lo que esto no es recomendable. De esta manera, se proponen 5 conjuntos difusos tipo triangular para cada variable de entrada y una resolución de 8 bits para la función de membresía.

\begin{tabular}{|l|l|l|l|l|l|}
\hline Variables & Nombre & Resolución & $\begin{array}{l}\text { Función de } \\
\text { Membresía }\end{array}$ & Conjuntos & \multicolumn{1}{|c|}{$\begin{array}{c}\text { Tipo de } \\
\text { controlador }\end{array}$} \\
\hline Entradas & $\begin{array}{l}\text { Error, } \\
\text { derivada } \\
\text { del error }\end{array}$ & $\begin{array}{l}10 \text { bits } \\
(0-1023)\end{array}$ & Triangular & 5 & Mamdani \\
\hline Salidas & Voltaje & $\begin{array}{l}8 \text { bits } \\
(0-255)\end{array}$ & Triangular & 5 & \\
\hline
\end{tabular}

Tabla 1 Diseño del controlador

Fuente: Elaboración Propia

Para la implementación del controlador, las variables de entrada son el error (1) en la temperatura del agua del acuario y la derivada de este error (2). La primera variable de entrada permite conocer qué tan alejado se encuentra la temperatura del valor de referencia, que para este caso, se ha establecido en $25^{\circ} \mathrm{C}$ ya que es un acuario tropical. La segunda variable de entrada permite conocer la variación de este error, esto es:

$\operatorname{error}(x)=$ Temp. de referencia - Temperatura actual

derivada del error $(y)=$ error actual - error anterior

\section{Variables de entrada- salida del CLD}

Las variables de entrada del controlador son las que permiten monitorear el sistema con una frecuencia determinada. En la figura 2, se muestra el universo de discurso de las variables que se proponen con base a los parámetros que se deben mantener dentro de un acuario de tipo tropical. Para la primera de las variables de entrada figura 2(a), se muestran los conjuntos difusos, donde las variables lingüísticas propuestas son: Error Negativo Grande, Error Negativo Pequeño, Nulo, Error Positivo Pequeño, Error Positivo Grande, contenidas en el rango de $-11^{\circ} \mathrm{C}$ a $11^{\circ} \mathrm{C}$.
La segunda entrada al controlador es la derivada del error, contenidas en el rango de $6^{\circ} \mathrm{C}$ a $6^{\circ} \mathrm{C}$ grados, cuyas variables linguíísticas se muestran en la figura 2 (b) y son: Error Positivo Grande, Error positivo Pequeño, Nulo, Error Negativo Pequeño, Error Negativo Grand. La salida del controlador manipula el voltaje del actuador. En la figura 2(c), se muestras las variables lingüísticas que se utilizaron para la salida: Voltaje Muy Pequeño, Voltaje Pequeño, Voltaje Medio, voltaje Grande, Voltaje Muy Grande.

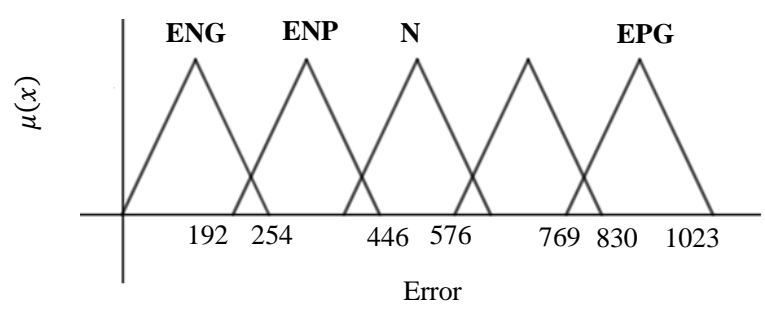

(a) Conjuntos difusos de entrada del error

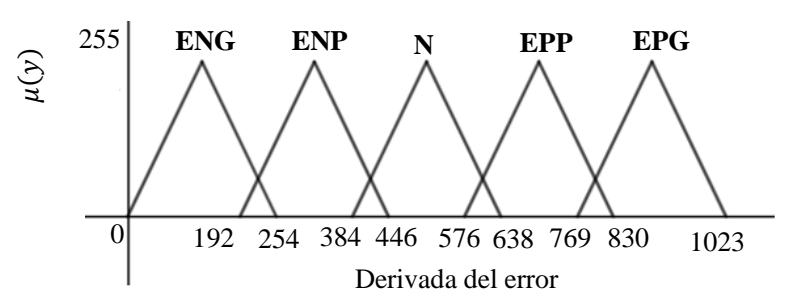

(b) Conjuntos difusos segunda entrada derivada del error

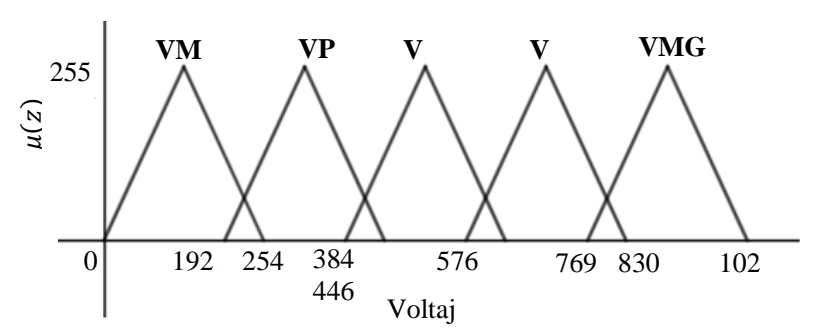

C) Conjuntos de salida Voltaje

Figura 2 Conjuntos difusos del controlador Fuente: Elaboración Propia

\section{Diseño del CLD}

\section{Fuzificación}

El primer paso del diseño del controlador es la Fuzificación, se propone como primer diseño el uso de funciones de tipo triangular debido a que proporciona cálculos más rápidos dentro de la configuración de hardware de la FPGA. Para obtener la función triangular de cada conjunto propuesto, se generan las tablas de búsqueda, empleando funciones triangulares, las cuales con base a (3), representan una función para cada uno de los conjuntos difusos

DE LA CRUZ-ALEJO, Jesús \& LOYA-ESCALANTE, Maria Teresa. Diseño de un control difuso tipo Mamdani para regular la temperatura de una Acuario tipo tropical. Revista del Diseño Innovativo. 2019 


$$
\mu(x)=\left\{\begin{array}{l}
\frac{a(b-x)}{b-c}, b \leq x \leq c \\
\frac{a(d-x)}{d-c}, c \leq x \leq d
\end{array}\right.
$$

Estas contienen los valores de membresía de todas las funciones triangulares del universo de discurso. El algoritmo que se implemento es con base a (4).

if $x<d$ then

$\mu(x)=\frac{[a(b-x)]}{(b-c)} \quad$ else

$\mu(x)=\frac{[a(d-x)]}{(d-c)}$

En la tabla 2, se muestra el rango de cada junto, donde se puede observar que son conjuntos simétricos, y el máximo traslape entre ellos es de dos conjuntos sin superar el punto medio de cada conjunto.

\begin{tabular}{|l|l|l|l|l|}
\hline \multicolumn{1}{|c|}{ Conjunto } & \multicolumn{1}{c|}{ Intervalo } & \multicolumn{1}{c|}{ Error } & \multicolumn{1}{c|}{$\begin{array}{c}\text { Derivada del } \\
\text { error }\end{array}$} & Punto medio \\
\hline ENG & $0-254$ & -11 a -6 & -6 a -3 & 127 \\
\hline ENP & $192-446$ & -8 a -2 & -4 a -1 & 319 \\
\hline N & $384-638$ & -3 a 3 & -1.5 a 1.5 & 511 \\
\hline EPP & $576-830$ & 2 a 8 & 1 a 4 & 703 \\
\hline EPG & $769-1023$ & 6 a 11 & 3 a 6 & 897 \\
\hline
\end{tabular}

Tabla 2 Tabla de los conjuntos

Fuente: Elaboración Propia

En la figura 3, se presentan los resultados de simulación obtenidos con el software dentro de la simulación del ISE donde $x, y$, representan la variable de entrada del error, y la derivada del error, respectivamente. Mientras que $m A$... $m E$ representan a las funciones de membresía para $x$, y $n A, \ldots$, las funciones de membresía para $y$. En la figura 3 , se observa que para valores distintos de $x$ y $y$, se activan los valores de membresías correspondientes a cada conjunto activado. Así por ejemplo, si $x=380$ y $y=150$, se obtiene un valor de membresía $m b=132$ y $n a=208$.

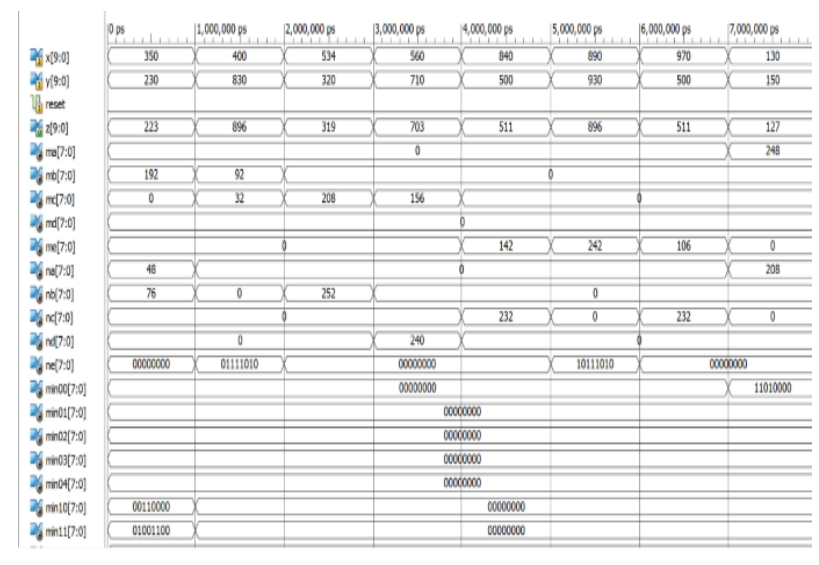

Figura 3 Simulación de la Fuzificación Fuente: Elaboración Propia

\section{Inferencia}

Se plantean las reglas de conocimiento que requiere el sistema, estas reglas son del tipo modus ponens (si- entonces) con base a (5).

If (antecedente) and (antecedente) then (consecuente)

Se genera, una matriz que contiene todas las comparaciones entre los valores de membresía generados por ambas entradas, como se muestra en la tabla 3, obtenidas con base a (6).

if $x$ es $A_{1}$ and $y$ es $B_{1}$ then $z=(1,1)$
if $x$ es $A_{1}$ and $y$ es $B_{2}$ then $z=(1,2)$
if $x$ es $A_{5}$ and $y$ es $B_{5}$ then $z=(5,5)$

Tabla 3 Matriz de inferencia

En la tabla 4, se muestra la Matriz de Asociación Difusa "FAM", en la cual se establecen las reglas de conocimiento que nos permitirán obtener una acción de control adecuada. Se puede observar que para un error positivo pequeño y una derivada del error positivo grande se obtiene una acción de control de voltaje grande.

\begin{tabular}{|c|c|c|c|c|c|c|}
\hline \multirow{7}{*}{ 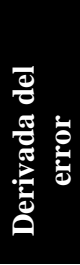 } & & \multicolumn{5}{|c|}{ Error } \\
\hline & & ENG & ENP & $\mathbf{N}$ & EPP & EPG \\
\hline & ENG & VMP & VMP & VMP & VMP & VMP \\
\hline & ENP & VMP & VMP & VMP & VMP & VMP \\
\hline & $\mathbf{N}$ & VMP & VMP & VMP & VMP & VMP \\
\hline & EPP & VMG & VG & VM & VG & VMG \\
\hline & EPG & VMG & $\mathrm{VG}$ & VM & $\mathrm{VG}$ & VMG \\
\hline
\end{tabular}

Tabla 4 Matriz de Asociación Difusa (FAM)

Fuente: Elaboración Propia

\section{Agregación}

Para llevar a cabo la etapa de agregación, se utiliza la implicacion máx, que representa la unión de las reglas difusas activadas y los conjuntos difusos que tiene un valor distinto de cero. El proceso se lleva a cabo a partir de la matriz obtenida en la etapa de inferencia. 
Para la etapa de agregación, las variables de entrada son los 25 mínimos generados en la etapa anterior $(\min 00, \ldots, \min 44)$. Como variables de salida, se obtiene un vector de agregación con los valores máximos de cada una de las columnas que representan los conjuntos difusos de la variable de salida (voltaje).

Posteriormente, se selecciona la primera columna de la matriz, la cual representa el primer conjunto difuso de la variable de salida etiquetada como voltaje muy pequeño (VMP), la cual, consta de los mínimos $\min 00, \min 10, \min 20, \min 30, \min 40$, obteniéndose el máximo valor de ella, con base a (7).

$\operatorname{Max} \_C 1=\max (([(\min 00, \min 10), \max (\min 20, \min 30)], 40)(7)$

El resultado final es un vector que contiene los máximos de cada una de las columnas, el cual se obtiene mediante (8):

$$
\mu\left(z_{i}\right)=\max \left[R_{1, i} \ldots R_{5, i}\right]
$$

\section{Defuzificación}

El método propuesto en este trabajo es el de niveles alfa obtenido mediante (9). Este método convierte la acción de control difusa de salida a sus correspondientes valores reales. En la figura 4, se muestra el código desarrollado en VHDL, para el cual se utilizo el ciclo if-then.

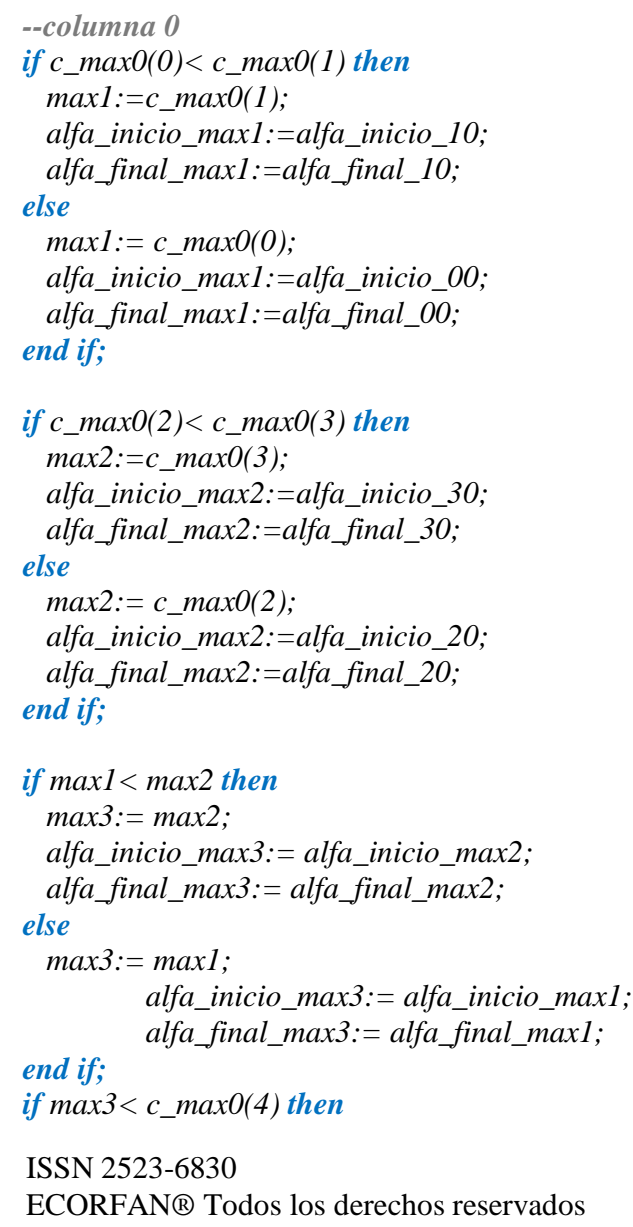

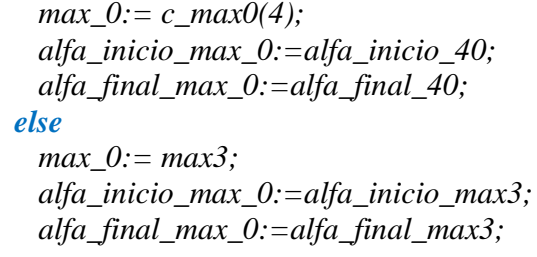

Figura 4 Agregación

niveles alfa $=\frac{\sum_{k=1}^{N}\left(\frac{x_{f}^{\alpha_{k}-\alpha_{i}^{\alpha_{k}}}}{2}\right)+x_{i}^{\alpha_{k}}}{N}$

Donde $x_{f}^{\alpha_{k}}$ es nivel alfa final, $x_{i}^{\alpha_{k}}$ es nivel alfa inicial, del valor de membresía que se obtiene después de haber llevado a cabo la etapa de agregación, $N$ es el número de conjuntos difusos de salida que tienen un grado de pertenencia distinto de cero.

\section{Caracterización del sistema}

Para llevar a cabo la acción de control y poder activar los actuadores (calentador y ventilador), se hace de un condicional If-then dentro de la programación en VHDL. En primera instancia, se realiza un análisis con base a las reglas de control propuestas y se propone controlar cada actuador partiendo del punto de equilibrio que en nuestro caso es un valor digital de 512 que representa un voltaje de $1.43 \mathrm{~V}$, que es le punto de equilibrio de la señal PWM.

Con base a los conjuntos del voltaje de salida (VMP, VP, VM), si la acción de control está dentro del rango de 0 a 512 , se acciona el ventilador, ya que con base al análisis realizado se determina que la temperatura del acuario es alta, esta acción de control se modula a través de la señal PWM en un rango de 32 posiciones.

Por otro lado, si la acción de control es mayor a 512, en los conjuntos de la variable de salida voltaje (VM, VG, VMG), se acciona el calentador, ya que con base al análisis realizado se determina que la temperatura del acuario es baja, por lo que se requiere controlar la temperatura del agua en el acuario. En la figura 5, se muestra el algoritmo de la acción de control. 
Revista del Diseño Innovativo

Septiembre, 2019 Vol.3 No.8 1-9

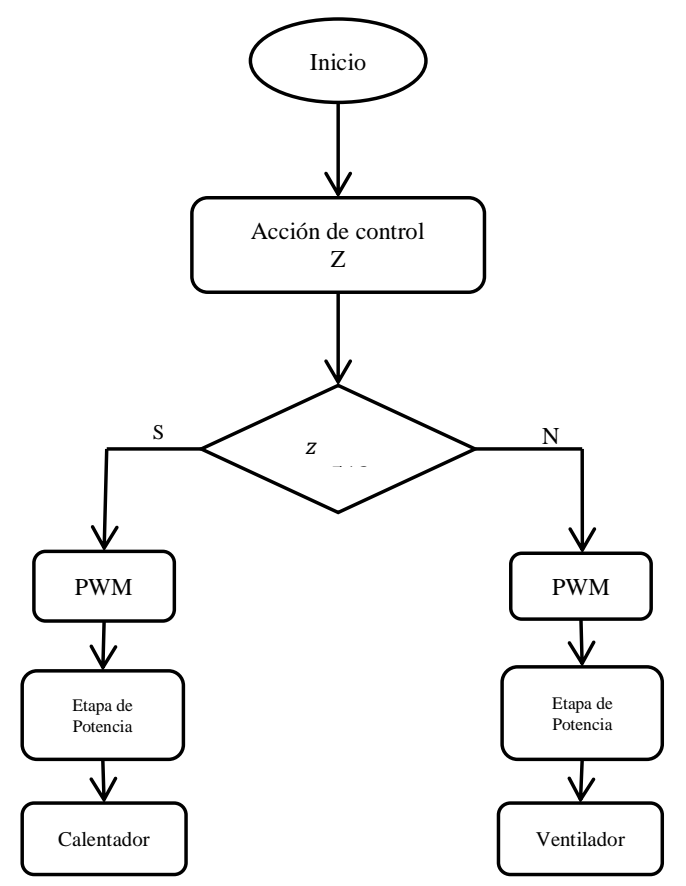

Figura 5 Caracterización de la variable de salida Fuente: Elaboración Propia

\section{Resultados de simulación y experimentales}

Para realizar la validación de los resultados, se tomó una muestra de 50 lecturas, los resultados experimentales del controlador se muestran en la tabla 5, donde se puede observar que la diferencia entre los resultados obtenidos de la implementación en la FPGA utilizando el método de niveles alfa y los obtenidos usando Matlab, es mínima.

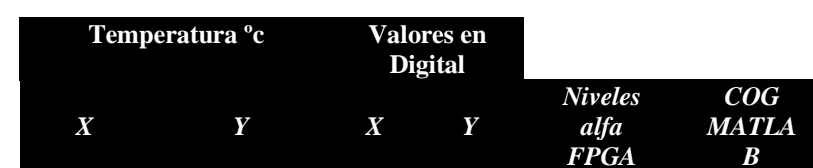

\begin{tabular}{|r|r|r|r|r|r|}
\hline 19,0537634 & 19,16129032 & 5 & 15 & 127 & 128 \\
\hline 19,1075269 & 20,07526882 & 10 & 100 & 127 & 128 \\
\hline 19,2258065 & 19,95698925 & 21 & 89 & 127 & 127 \\
\hline 19,3225806 & 28,67741935 & 30 & 900 & 896 & 895 \\
\hline 19,3870968 & 19,83870968 & 36 & 78 & 127 & 127 \\
\hline 19,483871 & 19,53763441 & 45 & 50 & 127 & 127 \\
\hline 19,5376344 & 27,60215054 & 50 & 800 & 799 & 801 \\
\hline 19,7204301 & 20,34408602 & 67 & 125 & 127 & 127 \\
\hline 19,9677419 & 19,48387097 & 90 & 45 & 127 & 127 \\
\hline 20,1827957 & 20,93548387 & 110 & 180 & 127 & 127 \\
\hline 20,3225806 & 21,51612903 & 123 & 234 & 223 & 254 \\
\hline 20,3978495 & 20,61290323 & 130 & 150 & 127 & 127 \\
\hline 20,827957 & 25,37634409 & 170 & 593 & 607 & 566 \\
\hline 20,9354839 & 22,76344086 & 180 & 350 & 319 & 319 \\
\hline 21,1505376 & 22,22580645 & 200 & 300 & 319 & 319 \\
\hline 21,2580645 & 23,83870968 & 210 & 450 & 511 & 511 \\
\hline 21,3655914 & 27,70967742 & 220 & 810 & 799 & 822 \\
\hline 21,4193548 & 25,93548387 & 225 & 645 & 703 & 703 \\
\hline
\end{tabular}

ISSN 2523-6830

ECORFAN® Todos los derechos reservados

\begin{tabular}{|c|c|c|c|c|c|}
\hline 21,516129 & 25,09677419 & 234 & 567 & 511 & 511 \\
\hline 22,2258065 & 26,95698925 & 300 & 740 & 703 & 703 \\
\hline 22,7096774 & 20,61290323 & 345 & 150 & 127 & 127 \\
\hline 22,7634409 & 21,47311828 & 350 & 230 & 223 & 242 \\
\hline 22,8387097 & 27,84946237 & 357 & 823 & 799 & 868 \\
\hline 23,3010753 & 27,92473118 & 400 & 830 & 896 & 843 \\
\hline 23,3010753 & 25,55913978 & 400 & 610 & 607 & 615 \\
\hline 23,6344086 & 27,38709677 & 431 & 780 & 799 & 745 \\
\hline 23,6344086 & 26,07526882 & 431 & 658 & 703 & 703 \\
\hline 23,7311828 & 25,74193548 & 440 & 627 & 607 & 664 \\
\hline 23,8924731 & 23,64516129 & 455 & 432 & 415 & 463 \\
\hline 24,6989247 & 25,34408602 & 530 & 590 & 607 & 557 \\
\hline 24,7419355 & 22,44086022 & 534 & 320 & 319 & 319 \\
\hline 24,8387097 & 29,61290323 & 543 & 987 & 896 & 703 \\
\hline 25,0215054 & 26,6344086 & 560 & 710 & 703 & 703 \\
\hline 25,0967742 & 23,90322581 & 567 & 456 & 511 & 511 \\
\hline 25,1182796 & 29,04301075 & 569 & 934 & 896 & 703 \\
\hline 25,2365591 & 22,95698925 & 580 & 368 & 319 & 319 \\
\hline 25,9784946 & 22,70967742 & 649 & 345 & 319 & 319 \\
\hline 26,2903226 & 27,22580645 & 678 & 765 & 703 & 703 \\
\hline 26,5268817 & 23,83870968 & 700 & 450 & 511 & 511 \\
\hline 27,3870968 & 29,43010753 & 780 & 970 & 896 & 895 \\
\hline 28,0322581 & 24,37634409 & 840 & 500 & 511 & 511 \\
\hline 28,5698925 & 29 & 890 & 930 & 896 & 895 \\
\hline 28,5698925 & 26,29032258 & 890 & 678 & 896 & 703 \\
\hline 28,6451613 & 25,89247312 & 897 & 641 & 896 & 703 \\
\hline 29,4301075 & 24,37634409 & 970 & 500 & 511 & 511 \\
\hline 29,4516129 & 25,93548387 & 972 & 645 & 896 & 703 \\
\hline 29,5376344 & 20,61290323 & 980 & 150 & 127 & 127 \\
\hline 29,6451613 & 29,75268817 & 990 & 1000 & 896 & 895 \\
\hline 29,7311828 & 29,98924731 & 998 & 1022 & 896 & 895 \\
\hline 29,7634409 & 19,30107527 & 1001 & 28 & 127 & 127 \\
\hline 30 & 29,64516129 & 1023 & 990 & 0 & 512 \\
\hline
\end{tabular}

Tabla 5 Resultados experimentales del controlador Fuente: Elaboración Propia

En el gráfico 1(a), se muestra la comparación de los resultados experimentales en color azul y los obtenidos en Matlab en color rojo. Se puede observar estadísticamente que la media de Niveles alfa es de 510.62 y la obtenida por Matlab es de 510.82, obteniendo un error de aproximación de $2.94 \%$. Este porcentaje es aceptable, el cual, se obtuvo con base a (10).

$$
\begin{aligned}
& \text { Error }=\frac{\delta_{\text {niveles alfa }}-\delta_{\operatorname{cog}}}{\text { resolución }} * 100 \\
& \text { Error }=\frac{302.43-272.32}{1023} * 100=2.94 \%
\end{aligned}
$$

En 1(b) se muestran la desviación de los resultados obtenidos usando una campana de gauss. 
Otro resultado que se puede obtener a partir de las reglas de conocimiento propuesta es la superficie de las reglas de control que se muestra en el gráfico 2(a), la cual, presenta los resultados obtenidos en Matlab, mientras que en (b), la superficie obtenida a partir de los resultados obtenidos por el controlador implementado en la FPGA.

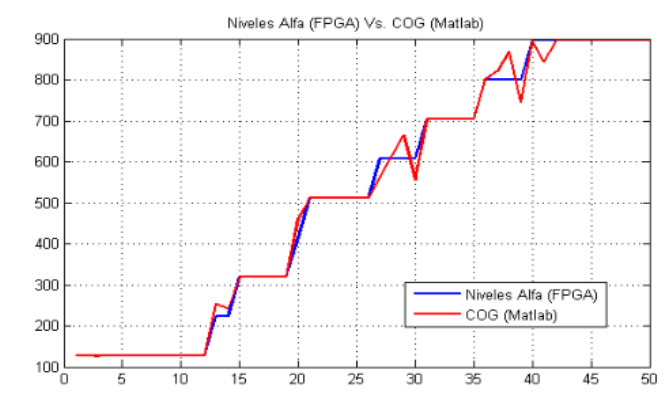

(a)

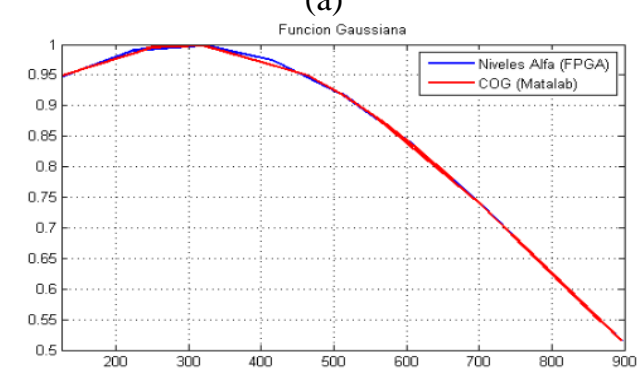

(b)

Gráfico 1 Análisis estadístico COG vs Niveles Alfa Fuente: Elaboración Propia

La similitud entre ambas, nos muestra la precisión de los resultados, obteniéndose una función monótona ya que su acción se realiza suavemente. En la figura 6, se muestran los diferentes ciclos de trabajo que se implementaron en la tarjeta FPGA.

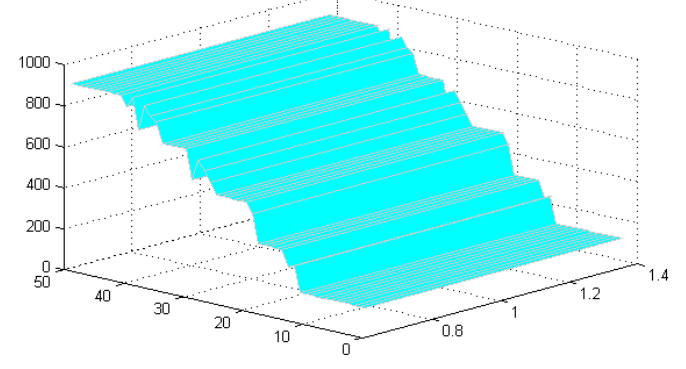

(a) $\mathrm{COG}_{\text {Matlab }}$

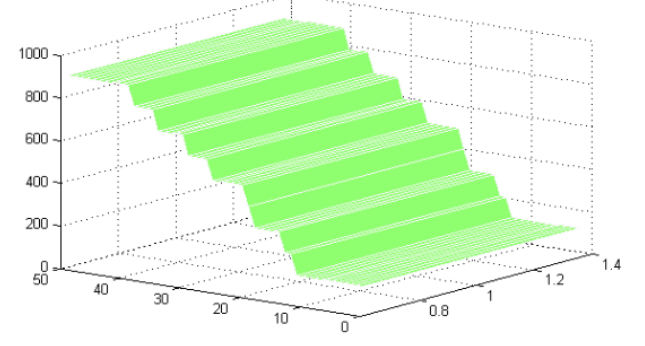

(b) Niveles alfa ${ }_{\mathrm{FPGA}}$

Gráfico 2 Superficies de control

ISSN 2523-6830

ECORFAN® Todos los derechos reservados
Como se observa, conforme aumenta el porcentaje del ciclo de trabajo, aumenta el tiempo que se mantiene encendido, variándose con esta la potencia de la resistencia y la velocidad del ventilador.

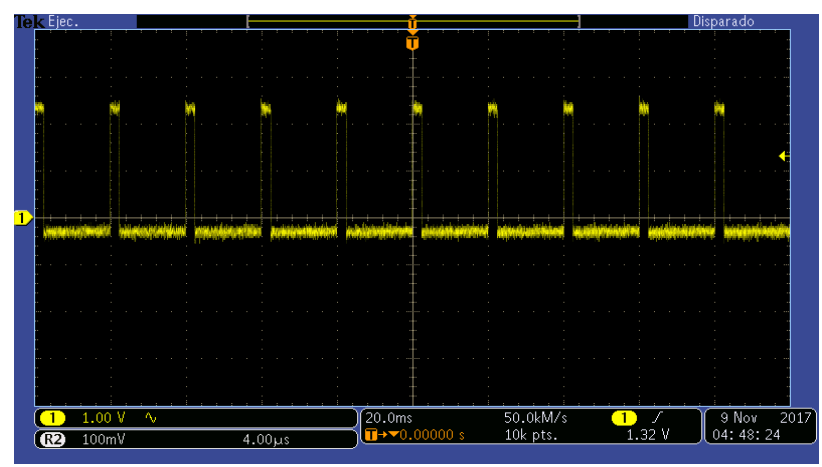

(a) $6.15 \%$ Ciclo de trabajo

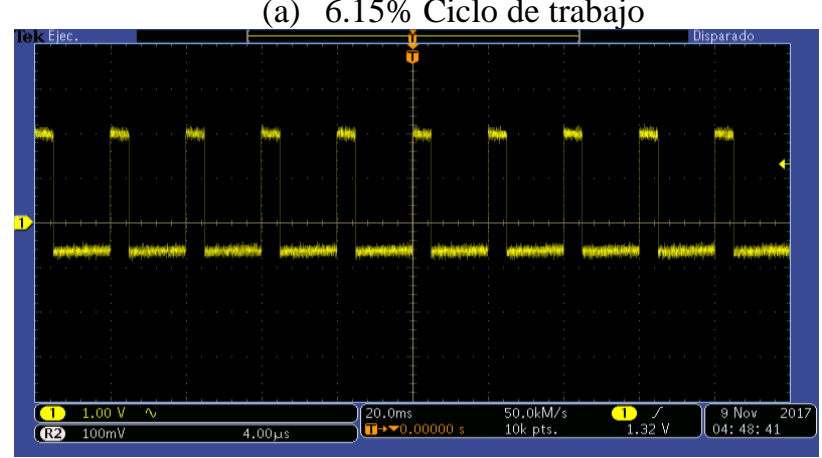

(b) $12.41 \%$ ciclo de trabajo

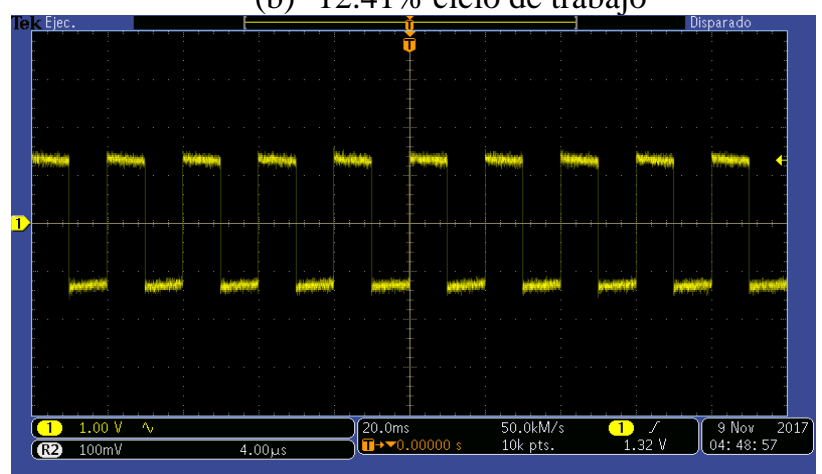

(c) $24.92 \%$ ciclo de trabajo

Figura 6 Señal PWM

Fuente: Elaboración Propia

En el gráfico 3, se muestra la respuesta del calentador ante los cambios que se presentan por la acción de control, donde se observa que la corriente del calentador responde de manera lineal con respecto a los cambios generados por la señal PWM.

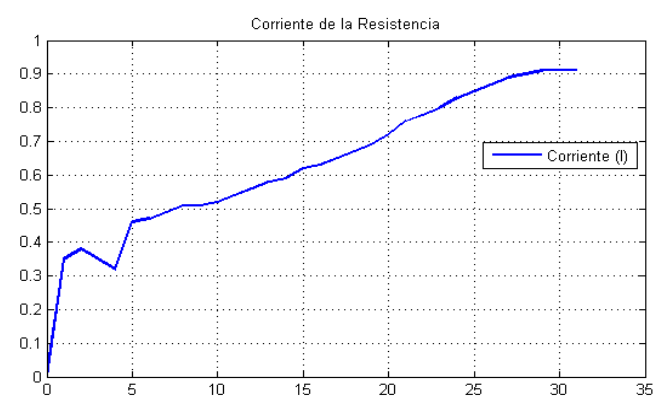

Gráfico 3 Respuesta del calentador

DE LA CRUZ-ALEJO, Jesús \& LOYA-ESCALANTE, Maria Teresa. Diseño de un control difuso tipo Mamdani para regular la temperatura de una Acuario tipo tropical. Revista del Diseño Innovativo. 2019 
En el gráfico 4, se muestra la respuesta del ventilador ante los cambios que se presentan por la acción de control, donde se observa que el voltaje del ventilador responde de manera lineal con respecto a los cambios generados por la señal de PWM. Después de haber diseñado el PWM y acoplarlo a los actuadores a través de una etapa de potencia se obtienen los resultados mostrados en la tabla 6 , donde se puede ver la acción de control sobre cada uno de los actuadores e ir variando de acuerdo al ciclo de trabajo establecido, por el controlador.

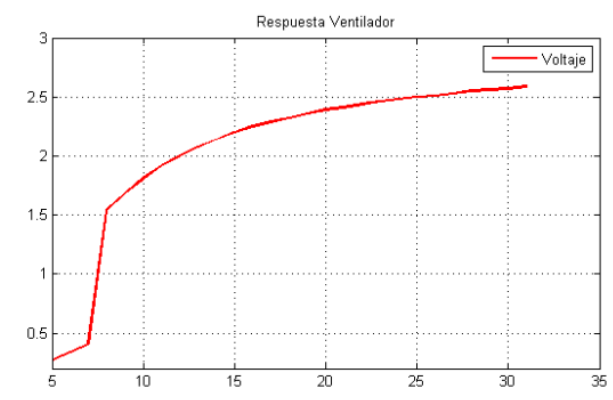

Gráfico 4 Respuesta del Ventilador Fuente: Elaboración Propia

La respuesta del sistema con base al controlador, presenta resultados favorables que se demuestran estadísticamente, obteniéndose un error del $2.94 \%$ muy aceptable. Tambien, se comprueba la funcionalidad del método propuesto con respecto al controlador diseñado en Matlab, así también se comprueba físicamente la respuesta del calentador hacia la repuesta de la señal PWM.

\begin{tabular}{|r|r|r|r|r|}
\hline Estados & \multicolumn{1}{c}{ Salida PWM (V) } & \multicolumn{1}{c|}{ Vout } & \multicolumn{1}{c|}{ calentador } & Ciclo de Trabajo (\%) \\
\hline $\mathbf{0}$ & 0 & 0 & 0 & 0 \\
\hline $\mathbf{1}$ & 0.9 & 0.34 & 0.35 & 3,125 \\
\hline $\mathbf{2}$ & 0.180 & 0.86 & 0.38 & 6,25 \\
\hline $\mathbf{3}$ & 0.270 & 0.146 & 0.35 & 9,375 \\
\hline $\mathbf{4}$ & 0.360 & 0.209 & 0.32 & 12,5 \\
\hline $\mathbf{5}$ & 0.450 & 0.273 & 0.46 & 15,625 \\
\hline $\mathbf{6}$ & 0.540 & 0.340 & 0.47 & 18,75 \\
\hline $\mathbf{7}$ & 0.629 & 0.407 & 0.49 & 21,875 \\
\hline $\mathbf{8}$ & 0.720 & 1.54 & 0.51 & 25 \\
\hline $\mathbf{9}$ & 0.809 & 1.68 & 0.51 & 28,125 \\
\hline $\mathbf{1 0}$ & 0.900 & 1.81 & 0.52 & 31,25 \\
\hline $\mathbf{1 1}$ & 0.990 & 1.92 & 0.54 & 34,375 \\
\hline $\mathbf{1 2}$ & 1.08 & 2.00 & 0.56 & 37,5 \\
\hline $\mathbf{1 3}$ & 1.17 & 2.08 & 0.58 & 40,625 \\
\hline $\mathbf{1 4}$ & 1.25 & 2.14 & 0.59 & 43,75 \\
\hline $\mathbf{1 5}$ & 1.35 & 2.20 & 0.62 & 46,875 \\
\hline $\mathbf{1 6}$ & 1.43 & 2.25 & 0.63 & 50 \\
\hline $\mathbf{1 7}$ & 1.52 & 2.29 & 0.65 & 53,125 \\
\hline
\end{tabular}

\begin{tabular}{|r|r|r|r|r|}
\hline $\mathbf{1 8}$ & 1.61 & 2.32 & 0.67 & 56,25 \\
\hline $\mathbf{1 9}$ & 1.70 & 2.36 & 0.69 & 59,375 \\
\hline $\mathbf{2 0}$ & 1.79 & 2.39 & 0.72 & 62,5 \\
\hline $\mathbf{2 1}$ & 1.88 & 2.41 & 0.76 & 65,625 \\
\hline $\mathbf{2 2}$ & 1.97 & 2.44 & 0.78 & 68,75 \\
\hline $\mathbf{2 3}$ & 2.06 & 2.46 & 0.80 & 71,875 \\
\hline $\mathbf{2 4}$ & 2.15 & 2.48 & 0.83 & 75 \\
\hline $\mathbf{2 5}$ & 2.242 & 2.50 & 0.85 & 78,125 \\
\hline $\mathbf{2 6}$ & 2.33 & 2.51 & 0.87 & 81,25 \\
\hline $\mathbf{2 7}$ & 2.42 & 2.53 & 0.89 & 84,375 \\
\hline $\mathbf{2 8}$ & 2.51 & 2.55 & 0.90 & 87,5 \\
\hline $\mathbf{2 9}$ & 2.60 & 2.56 & 0.91 & 90,625 \\
\hline $\mathbf{3 0}$ & 2.69 & 2.57 & 0.91 & 93,75 \\
\hline $\mathbf{3 1}$ & 2.78 & 2.59 & 0.91 & 96,875 \\
\hline
\end{tabular}

Tabla 6 Respuesta del sistema Fuente: Elaboración Propia

\section{Conclusiones}

El uso de dispositivos FPGA permite mayor capacidad de procesamiento de información, además de cumplir con la función de acoplar los elementos del sistema referenciado en la lógica del controlador difuso desarrollado. Tambien, se pudo observar que la dependencia o variación de la potencia del calentador por la cantidad de agua en el acuario se mantiene, y se controla su potencia a través de la acción de control mediante la señal PWM, por lo que no habría problemas si se colocara un calentador con mayor potencia que se tiene en función de los litros de agua, debido a que la referencia de la temperatura del agua del acuario, se regula a través de la potencia del calentador y de esta manera, se mejora la vida de los peces tropicales a través del control difuso de la temperatura aplicado a el ecosistema de una acuario, evitando totalmente el descuido por el control manual. Los resultados de simulación y experimentales confirman las predicciones teóricas.

\section{Referencias}

I. M. Mariani, El gran libro de los peces, Planeta

II. M. A. Olmos Castillo, «Tutorial de Introduccion de Lógica Borrosa,» 2008. [En línea]. Available: http://www.dma.fi.upm.es/recursos/aplic aciones/logica_borrosa/web/tutorial_fuz zy/introduccion2.html.

III. Atlas ilustrado del acuario, Susaeta. 
IV. Guia de Acuariofilia. Manual de un hobby fascinante, EHEIM

V. J. Lloret Pineda, «Diseño, instalación y mantenimiento de unacuario de exposición de agua dulce.,» Cádiz, 2006.

VI. D. Guzman y V. Castaño, «La Logica Difusa en Ingenieria: principios, Aplicaciones y Futuro,» Ciencia y tecnologia: Investigación, 2006.

VII. F. Rodriguez, Acuariofilia Rentable. Manual teorico-practico para la cria de peces.

VIII. H. A. Caicedo Analuisa y D. S. Obando Panchi , «Diseño e implementación de un sistema automatizado para el control del acuario del museo de Ciencias de la escuela politecnica Nacional,» Quito, Ecuador, 2011.

IX. L. A. Sala Carllosama y A. P. Arcos Lara, «Construccion de un prototipo de deteccion y control electronico de temperatura e ilumnicacion para el ecosistema de un acuario de peces tropicales en un clima templado.,» Peru, 2014.

X. H. Nelson Acosta y J. Garrido Salas, Diseño de controladores dedicados a la logica difusa, Madrid, España, 2006.

XI. P. Ponce Cruz, Inteligencia Artificial con aplicaciones a la Ingeniera, Mexico: Alfaomega, 2010

XII. W. Banks y G. Hayward, fuzzy Logic in Embedded Microcomputer and control System, Byte Craft, 2002

XIII. D. G. Maxinez y J. Alcalá, VHDL, el arte de programar sistemas digitales, Monterrey, México: CECSA, 2002

XIV. J. C. Garcia Montalva, J. De la Cruz Alejo y J. Diaz Salgado, «Fuzzy Logic Control on FPGA Using LabVIEW,» Multibody, Mechatronic Systems, pp. 261-270, 2014

XV. R. Antonio Mendez, J. De la Cruz Alejo y O. Peñaloza Mejia, «Fuzzy Logic Control on FPGA for Solar Tracking System,» Multibody Mechatronic System, pp. 11-21, 2015
XVI. Diseño, Automatizacion y Control, al alcance para todos,» [En línea]. Available: http://softwareparaingenieros.com/xilinx -ise-14-7/. [Último acceso: noviembre 2017].

XVII. J. De la Cruz Alejo, R. Antonio Méndez y M. Salazar Pereyra, «Fuzzy Logic Control on FPGA for two axes solar tracking,» The Natural Computing Application, 2017. 